\title{
Application of constrained optimization to radiotherapy planning
}

\author{
Otto A. Sauera) \\ Universität Würzburg, Klinik für Strahlentherapie, Josef-Schneider-Straße 4, 97080 Würzburg, Germany \\ David M. Shepard and T. Rock Mackie \\ University of Wisconsin, Medical School, Madison, Wisconsin 53706-1532
}

(Received 24 November 1998; accepted for publication 18 August 1999)

Essential for the calculation of photon fluence distributions for intensity modulated radiotherapy (IMRT) is the use of a suitable objective function. The objective function should reflect the clinical aims of tumor control and low side effect probability. Individual radiobiological parameters for patient organs are not yet available with sufficient accuracy. Some of the major drawbacks of some current optimization methods include an inability to converge to a solution for arbitrary input parameters, and/or a need for intensive user input in order to guide the optimization. In this work, a constrained optimization method was implemented and tested. It is closely related to the demanded clinical aims, avoiding the drawbacks mentioned above. In a prototype treatment planning system for IMRT, tumor control was guaranteed by setting a lower boundary for target dose. The aim of low complication is fulfilled by minimizing the dose to organs at risk. If only one type of tissue is involved, there is no absolute need for radiobiological parameters. For different organs, threshold dose, relative seriality of the organs or an upper dose limit could be set. All parameters, however, were optional, and could be omitted. Dose-volume constraints were not used, avoiding the possibility of local minima in the objective function. The approach was benchmarked through the simulation of both a head and neck and a lung case. A cylinder phantom with precalculated dose distributions of individual pencil beams was used. The dose to regions at risk could be significantly reduced using at least seven ports of beam incidence. Increasing the number of ports beyond seven produced only minor further gain. The relative seriality of organs was modeled through the use of an added exponent to the dose. This approach however increased calculation time significantly. The alternative of setting an upper limit is much faster and allows direct control of the maximum dose. Constrained optimization guarantees high tumor control probability, it is computationally more efficient than adding penalty terms to the objective function, and the input parameters are dose limits known in clinical practice. (C) 1999 American Association of Physicists in Medicine.

[S0094-2405(99)02711-X]

Key words: intensity modulated radiotherapy (IMRT), optimization, objective function

\section{INTRODUCTION}

In order to avoid local recurrence, curative radiotherapy aims to eradicate all clonogenic tumor cells within a defined target volume. ${ }^{1}$ This has to be done without severely injuring adjacent organs. Intensity modulated radiation therapy (IMRT) provides the potential to simultaneously fulfill both of these requirements. ${ }^{2}$ Due to the large number of parameters to be considered, such as the fluence of individual pencil beams, conventional interactive optimization techniques are not possible. This has led to the concept of inverse treatment planning: The aim of the treatment has to be described using certain treatment goals, so that a mathematical optimization algorithm can find the parameters which lead to an optimum dose distribution. For each pencil or elementary beam the primary fluence, the source position, the beam direction in space, the beam energy, and the radiation modality could be treated as variables. ${ }^{3}$ In practice, however, the solution space is usually restricted such that only the primary fluence weights vary. This obtains the most therapeutic gain when compared to conventional treatment techniques. Optimization of beam directions is important for a small number of ports only. ${ }^{4}$ Different mathematical methods have been utilized in order to perform optimization. They can be divided into two groups: stochastic methods such as simulated annealing ${ }^{5,6}$ and deterministic methods such as linear or quadratic programming, used by most groups working on this subject. Although deterministic methods are faster, there is a risk that, depending on the start up values, the algorithm could converge only to a local minimum. However, Deasy could show that nonconvex objective functions occur only if dose-volume constraints are explicitly considered. ${ }^{7}$

The most important task is the definition of a sensible treatment goal. This is accomplished through building an objective function. It is evident that this function has to include radiobiological considerations of the irradiated tissue. A sigmoidal dose response curve was suggested by many authors, starting with Holthusen ${ }^{8}$ in 1936. Brahme and co-workers applied Poisson statistics in order to model sigmoidal dose response. ${ }^{9}$ Tissue may be characterized by the dose value $D_{50}$, the dose resulting in a probability of $50 \%$ for the effect and the dose response gradient $\gamma$ at the $D_{50}$ dose level. In addition, a method that accounts for inhomogeneities of the 
dose distribution must be applied. Källman and co-workers, suggested defining a relative seriality, or its opposite, a relative parallelity of an organ. ${ }^{10}$ A serial organ is damaged if one subvolume of that organ is damaged, while a totally parallel organ losses its complete functionality only if all of the subvolumes are damaged. The complex physiology of living organs, however, makes it difficult to adequately model all possible radiation effects, and the clinical data are rare and insecure. Another problem is the need to average between different patients with different radiosensitivities. ${ }^{11,12}$ The applicability of both steep theoretical and flat averaged dose response functions for an individual patient is questionable. If many assumptions and parameters are not well justified, it is better at present to use more simplistic models that require a smaller number of parameters in which we have more confidence. In addition, simpler models result in faster numerical calculation and they are able to converge to a global optimum. Various approaches have already been used: maximize the minimum dose to the target, minimize the maximum dose to the regions at risk, least squares or an equivalent procedure to fit dose to a prescribed distribution, total energy imparted to the target and risk volumes. ${ }^{3,5,13-17}$ All of these methods generally result in dose distributions superior to those from conventional dose distribution plans. The end point, however, does not necessarily correspond to the treatment goal for an individual patient. In some methods it is necessary to use importance factors with no direct correspondence to biological parameters. Others, due to the possibility of using too rigorous dose constraints, lead to infeasibility of the optimization problem.

In this work, we investigated a strategy where dose to points in the target is forced to lie between a lower and an upper limit. With the lower limit, tumor control probability is adjustable. Different bounds may be attributed to different target points, if required. The dose value to any point within a region at risk is minimized. With this strategy, a weighting between tumor control and normal tissue complication probability is avoided. If only one type of region at risk is accounted for, generally no parameters are necessary in order to specify the radiation response of that organ. A dose as low as possible is automatically achieved. Certainly, the tolerance value where complication probability starts to rise has to be known by the clinician in order to recognize plans where no curative treatment is possible. This case is demonstrated for a U-shaped target, similar to a head and neck target volume. For an organ of parallel type, it might be desirable to apply a seriality parameter, in order to maximize the region of low dose. Alternatively a simple dose response relation could be used. This is proportional to dose for low dose values and almost flat beyond a specified threshold dose. If more than one type of vulnerable regions are involved, relative sensitivity and seriality parameters could be applied. Alternatively upper limit constraints may be assigned to each region. This is demonstrated for an L-shaped target surrounded with vulnerable regions which correspond to lung and spinal cord. With these approaches sophisticated optimization algorithms can be used together with clinically known tissue parameters.

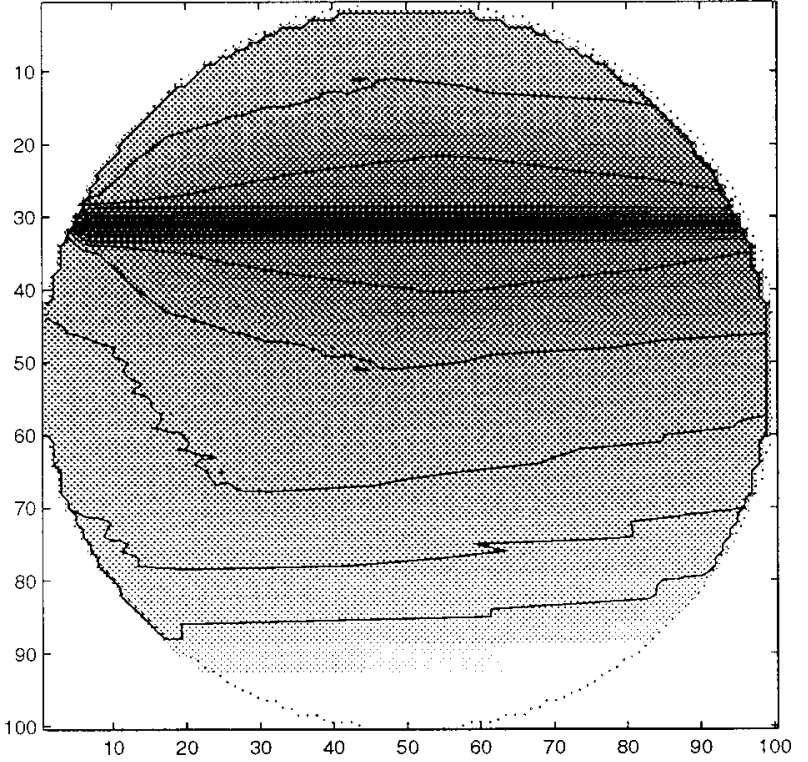

FIG. 1. Dose distribution from a pencil beam calculated with the convolution method. The cylinder phantom is $20 \mathrm{~cm}$ in diameter. The pixel size $2 \mathrm{~mm}$. To demonstrate the energy spread, the gray-scale is logarithmic.

\section{MATERIAL AND METHODS}

In order to test the proposed optimization strategy, a treatment planning system was written using the MATLAB5 programming language. ${ }^{18}$ It consists of easily replaceable modules for: (1) setting up geometry, beams, objective function, and constraints, (2) calculating pencil beam weights, and (3) evaluating and documenting results. Previous versions of the code had already been used to address a number of issues in radiation beam optimization. ${ }^{19,20}$

\section{A. Software modules and optimization algorithm}

The software follows the operations as needed for treatment planning. Regions of interest are entered as polygons and converted to binary matrices. The number of ports can vary. The gantry angles may be chosen manually or set equidistantly after input of a start value. In this work, for a number of ports greater than four, the automatic option was used. For a smaller number of ports the same rules as in conventional interactive treatment planning were applied.

The dose distribution from each pencil beam was precalculated. ${ }^{21}$ For this purpose a pencil beam of $2 \mathrm{MeV}$ primary photons with width of $2 \mathrm{~mm}$ and length of $110 \mathrm{~mm}$ was scanned over a water cylinder of $200 \mathrm{~mm}$ diameter. The dose distribution in the water cylinder was calculated for 100 positions of incidence, i.e., every $2 \mathrm{~mm}$ and stored in one hundred $100 \times 100$ matrices. For the dose calculation, the superposition method of Monte Carlo pregenerated energy deposition kernels was employed. Beam divergence was not included. An example of a pencil beam dose distribution is shown in Fig. 1. Due to cylindrical symmetry, the pencil beam dose kernels are invariant with respect to rotations 
around the central axis of the cylinder. Therefore, they can be used at any gantry angle. Only pencil beams potentially contributing to the target dose with a significant amount were used. It was set to $0.2 \%$ of the maximum dose of a pencil beam. The dose was calculated in one transversal plane only. For the purpose of evaluating different methods, this standardized phantom is of advantage. However, the system runs with any dose calculation engine.

The density of dose points used for the optimization may be chosen randomly. Our experience shows that a high density is necessary in regions where a high gradient of the dose distribution is desired. For the examples shown here a density of $80 \%$ for all regions of interest and $8 \%$ for the rest of the phantom was used.

For determination of the optimum fluence weights the MATLAB routine CONSTR is invoked. ${ }^{18}$ CONSTR is based on the method of Lagrange multipliers. At a minimum point $\mathbf{x}^{*}$ of the objective function $O(\mathbf{x})$ the Kuhn-Tucker equation

$$
\nabla O\left(\mathbf{x}^{*}\right)+\nabla \lambda \mathbf{g}\left(\mathbf{x}^{*}\right)=0
$$

with

$$
\boldsymbol{\lambda} \mathbf{g}\left(\mathbf{x}^{*}\right)=0
$$

is fulfilled. $\mathbf{g}\left(\mathbf{x}^{*}\right)$ is the vector of constraint functions, $\boldsymbol{\lambda}$ the set of Langrange parameters. The Kuhn-Tucker equation is solved using sequential quadratic programming. This means the problem is approximated by a quadratic form at each iteration point. The quadratic subproblem is solved by using the active set strategy: only constraints not fulfilled at the particular iteration point are considered. A similar strategy was published by Hristov and Fallone. ${ }^{22}$

\section{B. Objective function and optimization parameters}

The objective function $O$ was constructed to minimize the dose to all points at risk. If there is only one kind of tissue, especially if it is of serial type, the objective function may be simply the sum of dose to all points at risk. However, to be able to account for differences in sensitivity $w$ and seriality $S$ the following function was implemented:

$$
O=\sum_{i=1}^{\mathrm{RP}} w_{i} D_{i}^{S_{i}} .
$$

$D_{i}$ is the dose, the exponent $S_{i}$ accounts for the relative seriality, $w_{i}$ is the sensitivity at risk point $i$, and RP is the number of risk points considered. To organs where even a small volume receiving a relatively high dose causes severe damage, a high $S$ has to be attributed. This can be seen as an approximation of the lower part of the sigmoidal dose response relation. Instead of $w$, it is more convenient to use threshold values $D_{\text {th }}$ for the occurrence of complications at organs at risk. $D_{\text {th }}$ and $w$ are connected by setting the product $D_{\text {th }}^{S}$ equal to the same value for different organs. By doing this, all dose points receiving just the accordant threshold dose are penalized by the same amount, while dose values above the threshold are penalized according to their seriality exponent. Setting the value of the $w D_{\text {th }}^{S}$ to 1 ,
$w=D_{\mathrm{th}}^{-S}$.

For a parallel type of tissue, if regions to save shall not, or cannot be identified, it might be desirable to have the dose below a certain level for as much volume as possible. This can be achieved by increasing the burden with dose to regions being anyway above the threshold for complications. This behavior may be modeled by setting the exponent $S$ $<1$ in Eq. (2). Alternatively the dose response may be simulated by setting $S=1$ and substituting the dose $D$ in Eq. (2) with the effect $E$, where

$$
\begin{aligned}
& E=D \text { for } D<D_{\text {th }} \\
& E=a\left(D-D_{\text {th }}\right) \text { for } D \geqslant D_{\text {th. }} .
\end{aligned}
$$

$a$ is a little greater than 0 , in order to guide the optimization procedure into the right direction. It was set to 0.1 in this work. Equation (4) approximates the upper part of the sigmoidal dose response relation.

For the target volume, only a minimum desired value is set. This is done by defining a lower limit matrix (LLM) for the entire phantom, where we set

$$
L L M=0.95 \text { for target points, } L L M=0 \text { elsewhere. }
$$

By scaling the dose distribution with the prescribed dose, this setting guarantees a high tumor control probability, without detailed knowledge of the dose response curve. Additionally an upper limit matrix (ULM) is defined. Although an upper limit in the target region is desirable for certain disease sites, this matrix is generally not necessary, because due to the minimization of dose to normal tissue, the occurrence of hot spots is unlikely. The main reason for setting an upper limit was the reduction of the solution space, thus speeding up the calculation. The setting used in this work, if not otherwise stated was

$\mathrm{ULM}=1.1$ for target points, $\mathrm{ULM}=0.95$ elsewhere.

The introduction of upper limits bears the risk that a solution might not be feasible, if the limits are too restrictive. This is due to physical laws, especially the depth dose curve. However, with very little experience, appropriate values can be set to avoid infeasibility. The upper limit matrix also opens the possibility to set upper boundaries for the dose to regions at risk. This boundary can be set to the tolerance value of serial organs.

\section{Documentation and evaluation of results}

In order to compare different results, besides dose distributions and dose-volume histograms (DVH), basic parameters of the dose distributions, like minimum, maximum, mean value, and standard deviation of the dose to a specific region of interest were calculated. The importance of a parameter is linked to the type of tissue under consideration. For an entirely serial organ, the maximum dose is the limiting factor, while for a parallel structured organ the radiobiological response is linked closer to the mean dose and its standard deviation. 


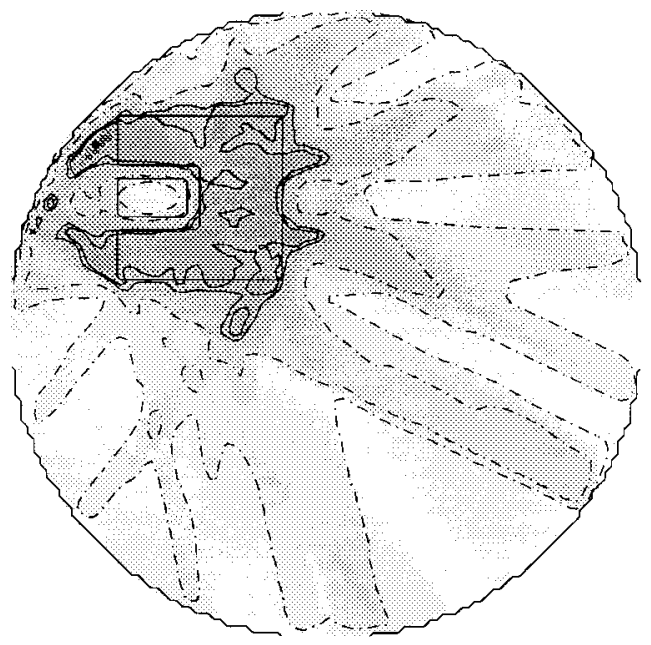

FIG. 2. Optimized dose distribution for a U-shaped target with a region at risk within its concavity. Seven incident ports with equidistant gantry angles were set. The lower boundary for target dose was 0.95 . Dose to the points at risk were minimized. Dash-dotted lines are the 0.2 and 0.5 isodose lines, the solid lines denote the 0.9 and 0.99 isodoses, respectively.

Considering two dose distributions having similar mean dose values, the one with the higher variance could have less vulnerable effect. This subject is controversial in the literature. The result of a recent survey from Kwa et al. ${ }^{23}$ is that mean dose predicts radiation pneumonitis, while for Zaider and $\mathrm{Amols}^{24}$ empirical data indicate that irradiating a partial volume of lung results in a lower complication rate than irradiating the whole organ uniformly. For eradication of a tumor, generally the minimum target dose is decisive. Additionally the quantity "equivalent uniform dose" (EUD) as proposed by Niemjerko was calculated. ${ }^{25}$ EUD is the dose resulting in the same probability of cell survival as the inhomogeneous dose distribution within the target volume,

$$
\mathrm{EUD}=-D_{0} \ln \left[\frac{1}{N} \sum_{i} e^{D_{i} / D_{0}}\right],
$$

where $D_{0}=1 / \alpha=-2 \mathrm{~Gy} / \ln \left(\mathrm{SF}_{2}\right)$ and $\mathrm{SF}_{2}$ is the survival fraction at $2 \mathrm{~Gy} . D_{i}$ is the dose at voxel $i$ and $N$ the number of voxels. In this work $\alpha=0.347 \mathrm{~Gy}^{-1}$, equivalent to $\mathrm{SF}_{2}$ $=0.5$ were used. For the mean dose, 70 Gy was assumed. For easier comparison EUD was normalized to 1 by dividing EUD with 70 Gy again. When the dose distribution is highly inhomogeneous the EUD tends to the minimum dose and when it is highly homogeneous EUD tends to the mean dose.

\section{RESULTS AND DISCUSSION}

To evaluate the proposed methods, they were applied to two cases related to clinical practice; a head and neck and a lung tumor treatment.

\section{A. Head and Neck case}

Treatment plans were calculated for a U-shaped target with a region at risk within its concavity (see Fig. 2). For the vulnerable tissue no radiobiological parameters were set. The dose was minimized for all points at risk. The number of

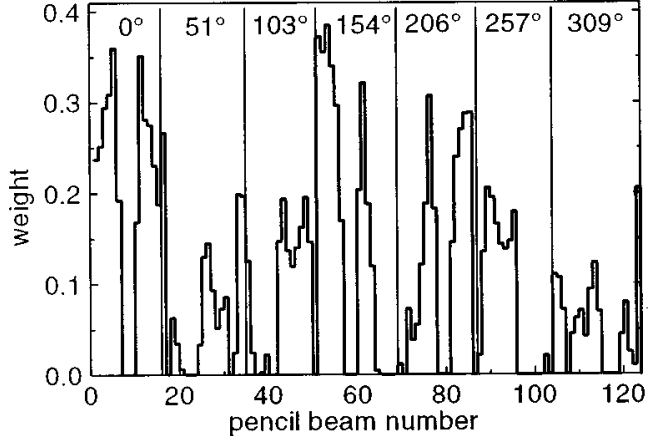

FIG. 3. Fluence profile corresponding to the dose distribution in Fig. 2. The gantry angles are indicated. $\left(0^{\circ}\right.$ is from left to right.)

incident ports with different gantry angles is varied from only one up to thirteen ports. Except for the case with the single port, the dose to the target is constrained between 0.95 and 1.1, Due to physical reasons, this is not possible if only one port is applied. Relaxing the upper limit, however, achieves a clinical meaningful solution even for this case. The dose distributions conform well with the target. This is shown for seven ports in Fig. 2 as an example. The fluence profiles for this dose distribution are plotted in Fig. 3. Only a few target voxels receive a dose less than 0.95 . The amount is controlled by an accuracy parameter for the limits. Here an accuracy of 0.01 is set, small enough not to compromise the tumor control probability. Additionally points not used for optimization may receive dose outside the specified limits. This is controlled by the point density used. For all points within the region at risk, a very low dose is achieved. Fig. 4 shows the corresponding dose volume histograms. With the knowledge of the dose necessary for the tumor and the risk of side effects, a safe treatment can be delivered.

Figure 5 shows the dependence of different parameters on the number of ports. For the number of ports equal or greater than two the target dose parameters are constant. The mean dose is a little above one, in the middle between the lower

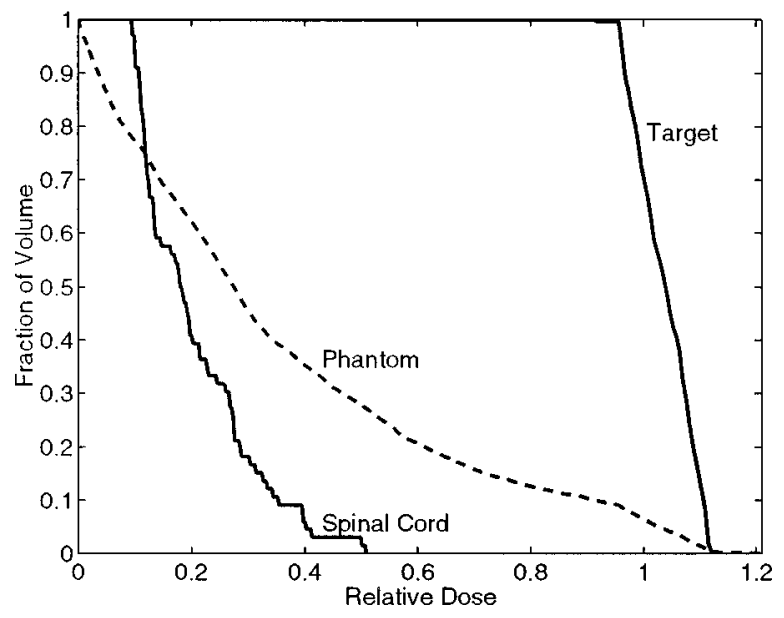

FIG. 4. Cumulated dose-volume histogram of the dose distribution in Fig. 2. Due to an accuracy parameter, a very small fraction of the target volume receives a dose less than the lower limit of 0.95 . The curve labeled "Phantom' ' is for the entire phantom. 

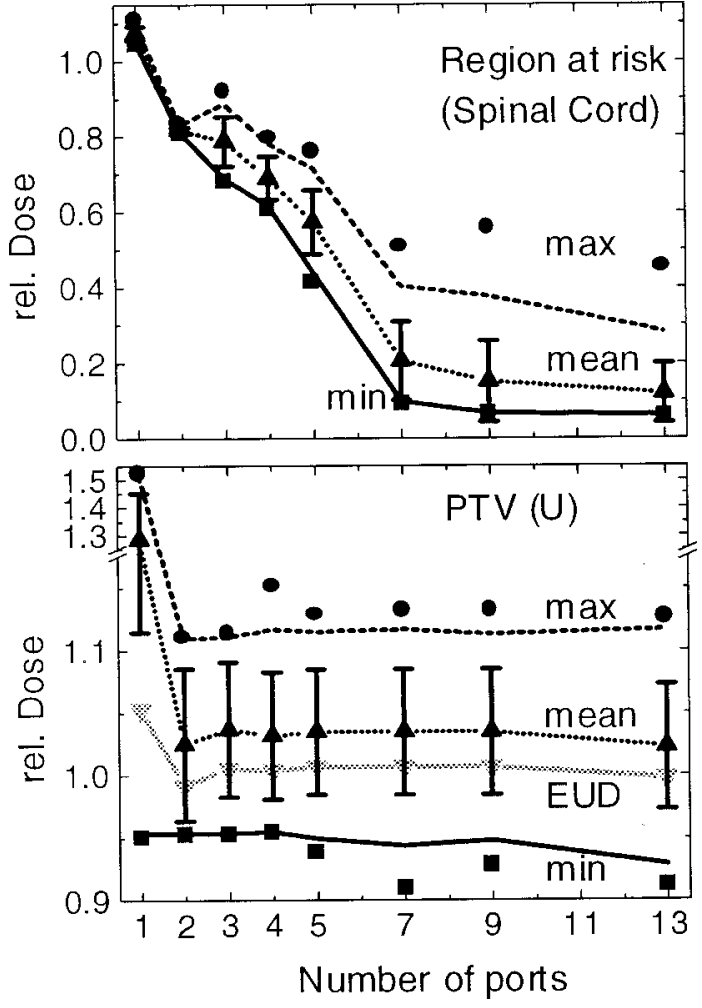

FIG. 5. Dependence of basic parameters of the dose distribution for the U-target case on the number of ports. Dashed and dotted lines near the points indicating maximum and minimum dose represent mean values for the 8 pixels with the highest and lowest dose, respectively.

and upper limits. This guarantees a EUD very close to the desired value of 1.0. All dose parameters for the region at risk are significantly decreasing with increasing number of ports. However, for a number of ports greater than seven the benefit is rather small. This is in agreement with findings from other authors. ${ }^{4,26}$

\section{B. Lung case}

In a more general case, the relative difference in the dose response properties of different tissues has to be taken into account. Therefore the optimization approach was tested on an L-shaped target with a region at risk of a more serial nature within its elbow. This setup mimics the spinal cord and is surrounded by a lung, an organ of parallel physiological structure (Fig. 6). The threshold dose for the lung was set to 0.3 , its seriality to 1 . For the spinal cord $D_{\text {th }}=0.5$ was used, and the seriality exponent $S$ was varied. Figures 6 and 7 show a dose distribution and a corresponding dose volume histogram for an example with seven ports and $S=8$ for the spinal cord. The behavior of the dose distribution with varying seriality exponent is summarized in Fig. 8. Increasing $S$ up to a value of 16 leads to a maximum dose for the organ at risk (risk 1, spinal cord) almost equal to the desired threshold value of 0.5. As seen in Fig.6, only very small regions at the corners of risk 1 receive a dose exceeding 0.5 . All dose parameters for risk 2 (lung) are almost independent of $S$ for the spinal cord and the mean dose is well below the limit of 0.3 .

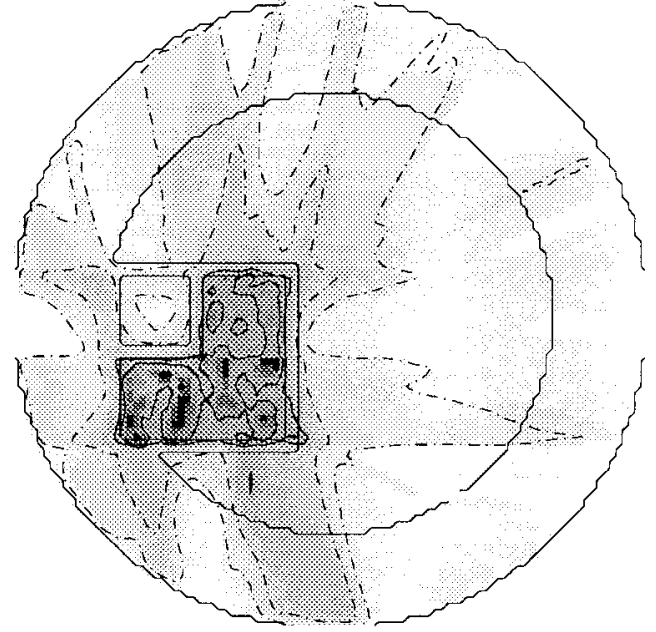

FIG. 6. Optimized dose distribution for the L-shaped target, surrounded by spinal cord and lung. Optimization was performed using Eqs. (1) and (2), with $S=8$ for spinal cord and $S=1$ for lung. $D_{\text {th }}$ values were 0.5 and 0.3 , respectively. Isodose lines as in Fig. 2.

Due to its position, around the target, the maximum dose to the lung is of the same order as target dose. The dose to the target itself is as enforced by the dose limit matrices. This approach eliminates the risk of "overcharging" the system, which would lead to infeasibility of the problem. Certainly, the end point of the optimization is determined not only by the radiobiological parameters $w$ and $S$ used here, but also by the geometry of the problem, and the technical and physical constraints and the degrees of freedom.

In order to investigate its gain, the optimization was performed for different numbers of ports set between one and thirteen (Fig. 9). Threshold dose values were set as previously, and $S=8$ was used as the seriality exponent for risk volume 1. Again, up to the number of four ports, the gantry angles were set manually. As expected, the main effect of increasing the number of ports is on risk 1 volume. Dose is

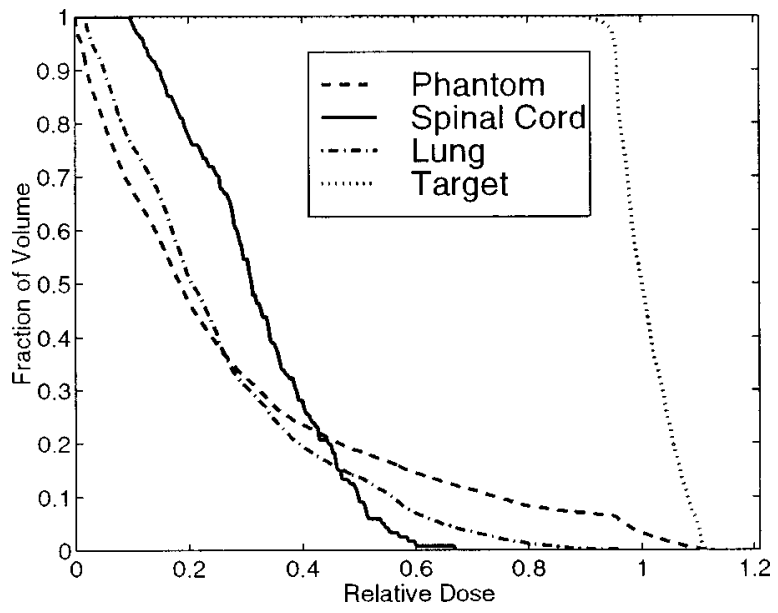

FIG. 7. Cumulated dose-volume histogram of the dose distribution in Fig. 5. Because of the high seriality set for the spinal cord, only a small volume receives a higher dose than the threshold set to 0.5 . The low seriality exponent for lung allows much of the volume to receive a dose higher than the threshold which was set to 0.3 . 

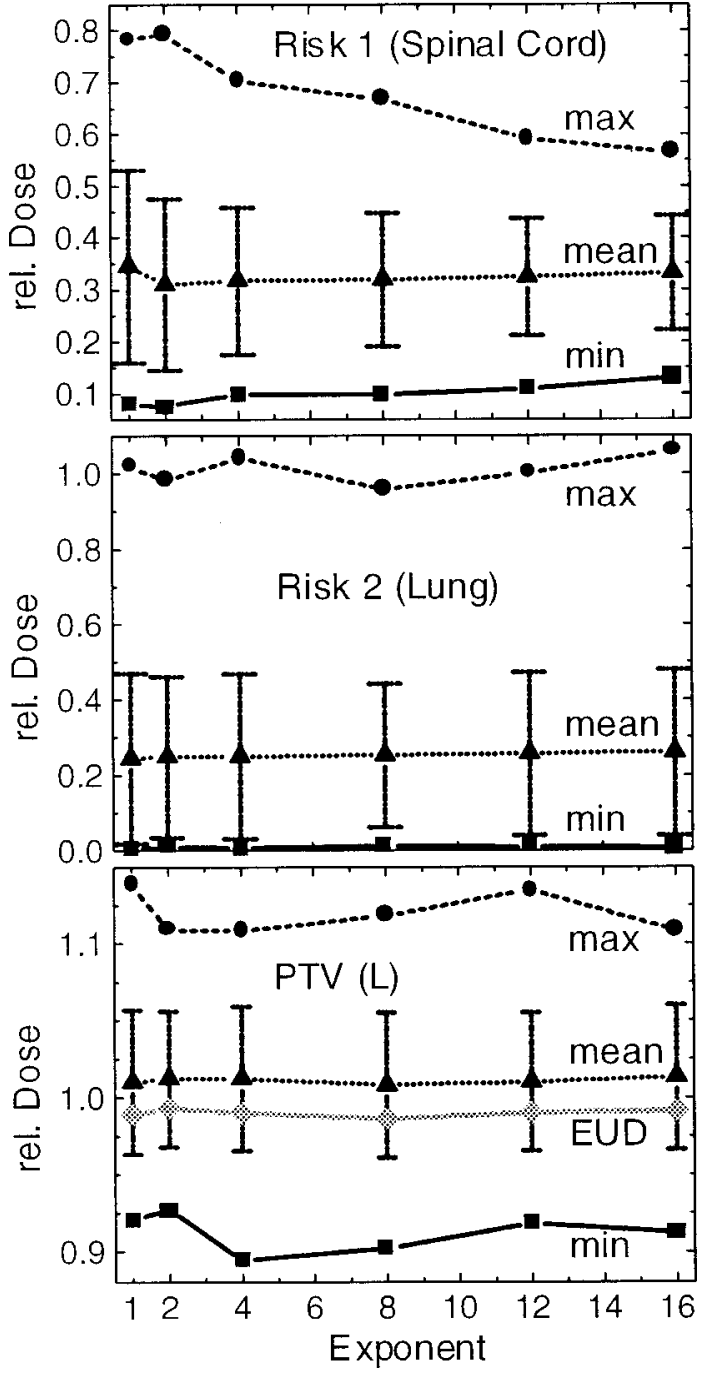

FIG. 8. Dependence of basic parameters of the dose distribution for the L-target case on the seriality exponent for spinal cord. The maximum dose for the spinal cord decreases with increasing exponent, while the values for the target and the lung remain nearly constant.

continuously decreasing, but for more than seven ports, the gain is very small. The decrease for the dose in risk 2 volume (lung) is small. While the standard deviation for three ports and more is rather constant, the mean dose is reduced from 0.3 for one port to 0.2 for thirteen ports.

Both results shown in Fig. 8 and in Fig. 9 indicate that the maximum dose, being determinative for the risk of injuring the spinal cord, is controllable by the seriality parameter. However, the control is not direct and increasing the exponent $S$ in Eq. (2), increases the computational burden. It is possible to control the maximum dose directly by setting upper limit values for each dose point within the region of interest. Having upper and lower limits with the lower limit greater than the upper limit at adjacent volumes, as for target and risk 1 in this example, involves the danger of not being able to reach a feasible solution. A strategy has to be applied in order to avoid such a situation. As shown in Fig. 10, for our test case, this problem occurs only if less than seven ports are applied. For less than seven ports, the upper limit
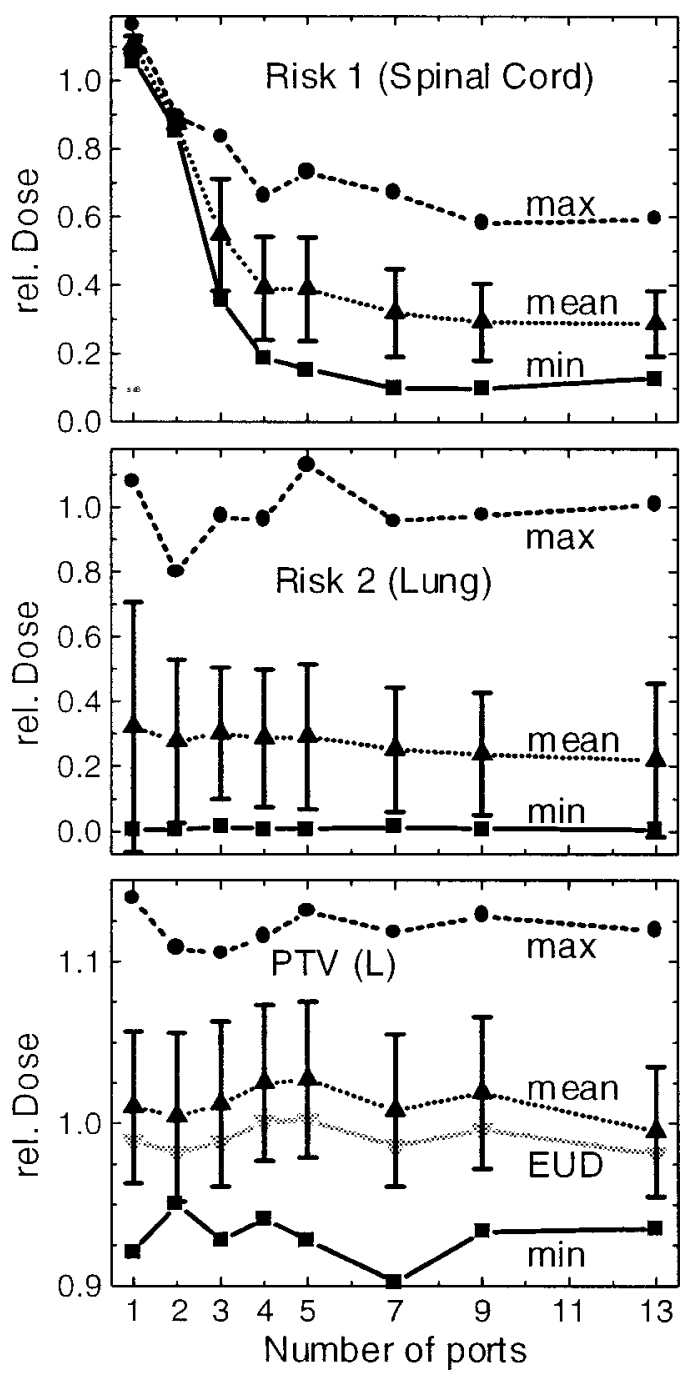

FIG. 9. Dependence of the basic parameters of the dose distribution for the L-target case on the number of ports. Input parameters are as in Fig. 5.

dose for risk 1 was increased, until a feasible solution is reached. The calculation time for this approach was an order of magnitude less than using an exponent of 8 . This confirms the advantage of using dose limit constraints directly. The overall CPU time needed for 100 pencil beams and $10^{4}$ dose points was about $4 \mathrm{~min}$ on a DEC $\alpha 4100$ computer. However, a big potential is left in order to speed up the calculation.

The capability to increase the low dose region within the lung is demonstrated in Fig. 11. According to Eq. (4), the increase of effect was reduced for dose values greater 0.5. Compared to the dose distribution from Fig. 6, the region of low dose is increased. This is paid with a higher dose to the high dose region. If a dose greater than 0.5 destroys the tissue anyway, this more inhomogeneous dose distribution should lead to a lower complication probability for the lung. A similar dose distribution was achieved using Eq. (2) with an exponent $S<1$.

\section{CONCLUSIONS}

We have implemented an optimization strategy which guarantees a high tumor control probability by simply setting 

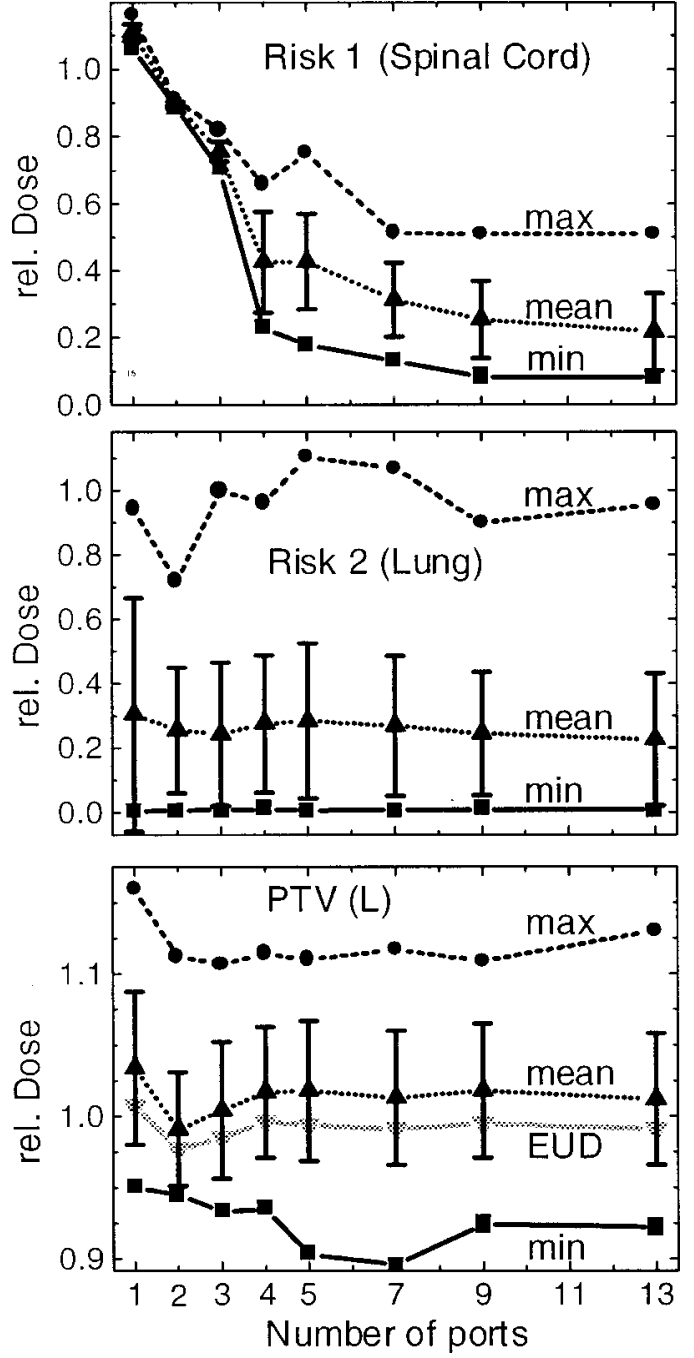

Fig. 10. Same as Fig. 9, but now $S=1$ for both lung and spinal cord. An upper limit was set for the spinal cord. This was 0.5 when the number of ports was greater than or equal to seven and as low as feasible for lower numbers.

a lower limit constraint on the target volume. Complication probability for organs at risk is controlled by minimizing the dose to that organ. It was shown that if only a serial type of vulnerable tissue has to be considered no radiobiological input parameter is necessary. If the parameters of the dose response curve for an individual patient's organ are not known with sufficient certainty, it is safer to keep the dose as low as technically achievable. For a number of tissue types being involved, radiobiological parameters generally have to be set in order to guide the optimization process. The number of parameters should be as small as possible, simple and closely linked to clinical experience. Our method sets a threshold dose causing damage to an organ and its relative seriality controlling the mean, the variance, and the maximum dose. These parameters are closely related to tolerance dose and volume effect conventionally used in clinical practice. An explicit dose-volume constraint, e.g., as used by Bortfeld, Stein, and Preiser is not necessary in our method. ${ }^{27}$ Dose and volume of too high dose for lung are minimized

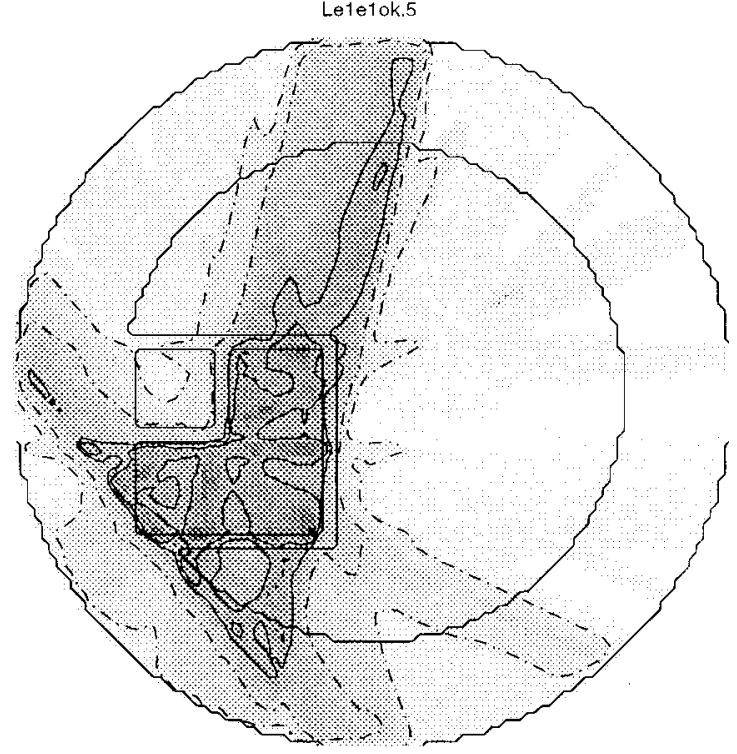

FIG. 11. Dose distribution achieved using Eq. (4). The increase of effect for the "lung" was set low for $D>0.5$. The upper limit for the "spinal cord" was 0.5 . Isodose lines as in Fig. 2.

automatically, avoiding possible infeasibility and computational problems due to the occurrence of multiple local minima if such constraints are set. ${ }^{7}$ To take advantage of the computational efficiency of modern optimization algorithms, it is desirable to use upper limit constraints for vulnerable regions. As demonstrated, this significantly improves the treatment plan and does not lead to infeasibility if used for small volumes of serial structure only. A similar tactic was used by Powlis et al. ${ }^{15}$ However, they were satisfied by finding a feasible solution by interactively changing the dose limits and did not attempt to further optimize the dose distribution.

The controversial issue of how many ports are required in order to improve uncomplicated tumor control (UTC) is discussed in the literature ${ }^{4,26}$ Our results confirm that in general more than five ports should be used. However the gain achieved with more than seven ports is small. Calculating UTC with very steep dose response curves may not result in a significant difference in UTC for three and seven ports. However, knowing the uncertainty in dose response of individual patients, the achievable reserve in tolerance should not be given away.

We have written a prototype of an "inverse treatment planning system" with the following features: The system minimizes dose to regions at risk, maintaining a minimum dose to target. The only parameter needed is the lower bound for target dose. The number of input parameters to specify vulnerable regions is optional. Corresponding to the complexity of the problem, no parameter or up to two parameters may be set, i.e., a threshold dose, a seriality exponent, or alternatively an upper bound. This makes the system flexible dependent on the knowledge of an individual case. Care was taken to avoid infeasibility. The system does not break down, even if only a single port is used, which seriously shrinks the 
ability to optimize the dose distribution. Optimization runs automatically, user interaction is not necessary. Only if upper limit constraints cannot be fulfilled, may the user wish to alter these settings. With an appropriate dose calculation engine the system developed may be used with real patients. Then, additionally technical constraints of the dose delivery device (such as a tomotherapy unit or a linear accelerator with multileaf-collimation) must be taken into account.

\section{ACKNOWLEDGMENTS}

O. S. was partly supported by Deutsche ForschungsGemeinschaft (Sa 481/2-1). D. S. was supported by NIH Grant No. CA48902.

\footnotetext{
a) Author to whom all correspondence should be addressed; electronic mail: sauer@mail.uni-wuerzburg.de

${ }^{1}$ T. R. Munro and C. W. Gilbert, "The relation between tumour lethal doses and the radiosensitivity of tumour cells," Br. J. Radiol. 34, 246251 (1961).

${ }^{2}$ A. Brahme, "Optimization of stationary and moving beam radiation therapy techniques,'” Radiother. Oncol. 12, 129-140 (1988).

${ }^{3}$ A. Gustafsson, B. K. Lind, and A. Brahme, "A generalized pencil beam algorithm for optimization of radiation therapy,' Med. Phys. 21, 343356 (1994).

${ }^{4} \mathrm{~S}$. Söderström and A. Brahme, "Which is the most suitable number of photon beam portals in coplanar radiation therapy?,' Int. J. Radiat. Oncol., Biol., Phys. 33, 151-159 (1995).

${ }^{5} \mathrm{~S}$. Webb, "Optimization by simulated annealing of three-dimensional, conformal treatment planning for radiation fields defined by a multileaf collimator,' Phys. Med. Biol. 37, 1689-1704 (1992).

${ }^{6} \mathrm{~S}$. Webb, “'Optimization of conformal radiotherapy dose distributions by simulated annealing,' Phys. Med. Biol. 34, 1349-1370 (1989).

${ }^{7}$ J. Deasy, "Multiple local minima in radiotherapy optimization problems with dose-volume constraints,' Med. Phys. 24, 1157-1161 (1997).

${ }^{8}$ H. Holthusen, "Erfahrungen über die Verträglichkeitsgrenze für Röntgenstrahlen und deren Nutzanwendung zur Verhütung von Schäden,' Strahlenther. Onkol. 57, 254-269 (1936).

${ }^{9}$ A. Brahme, "Treatment optimization using physical and radiobiological objective functions," in Medical Radiology, edited by A. R. Smith (Springer, Berlin, 1995), pp. 210-246.

${ }^{10}$ P. Källman, A. Ågren, and A. Brahme, "Tumour and normal tissue responses to fractionated non-uniform dose delivery,' 'Int. J. Radiat. Biol. 62, 249-262 (1992).
}

${ }^{11}$ D. J. Brenner, "Dose, volume and tumor-control predictions in radiotherapy,', Int. J. Radiat. Oncol., Biol., Phys. 26, 171-179 (1993).

${ }^{12} \mathrm{~S}$. Webb, "Optimum parameters in a model for tumour control probability including interpatient heterogeneity,', Phys. Med. Biol. 39, 18951914 (1994).

${ }^{13}$ T. Bortfeld, J. Bürckelbach, R. Boeseke, and W. Schlegel, "'Methods of image reconstruction from projections applied to conformation radiotherapy,', Phys. Med. Biol. 35, 1423-1434 (1990).

${ }^{14}$ T. Holmes and T. R. Mackie, " A filtered backprojection dose calculation method for inverse treatment planning," Med. Phys. 21, 303-313 (1994).

${ }^{15}$ W. D. Prowlis, M. D. Altschuler, Y. Censor, and E. L. Buhle, "Semiautomated radiotherapy treatment planning with a mathematical model to satisfy treatment goals,' Int. J. Radiat. Oncol., Biol., Phys. 16, 271-276 (1989).

${ }^{16} \mathrm{~J}$. Llacer, "Inverse radiation treatment planning using the Dynamically Penalized Likelihood method,' Med. Phys. 24, 1751-1764 (1997).

${ }^{17}$ S. V. Spirou and C.-S. Chui, "A gradient inverse planning algorithm with dose-volume constraints," Med. Phys. 25, 321-333 (1998).

${ }^{18}$ The Mathworks Inc., 24 Prime Park Way, Natick, MA, USA, MATLAB (1996).

${ }^{19}$ O. A. Sauer, D. M. Shepard, L. Angelos, and T. R. Mackie, “A comparison of objective functions for use in radiotherapy optimization," in Proceedings of the 12th International Conference on Computers in Radiation Therapy, edited by D. D. Leavitt and G. Starkschall (Medical Physics Publishing, Madison, WI, 1997), pp. 313-316.

${ }^{20}$ D. M. Shepard, L. Angelos, O. A. Sauer, and T. R. Mackie, "A simple model for examining radiotherapy optimization," in Ref. 19, pp. 323326.

${ }^{21}$ L. Angelos, D. M. Shepard, O. A. Sauer, and T. R. Mackie, "Data sets for studying radiotherapy optimization,', in Ref. 19, 480 pp. (abstr.).

${ }^{22}$ D. H. Hristov and B. G. Fallone, "An active set algorithm for treatment planning optimization,'” Med. Phys. 24, 1455-1464 (1997).

${ }^{23}$ S. L. S. Kwa, J. C. M. Theuws, A. Wagenaar, E. M. F. Damen, L. J. Boersma, P. Baas, S. H. Muller, and J. V. Lebesque, "'Evaluation of two dose-volume reduction models for the prediction of radiation pneumonitis,"' Radiother. Oncol. 48, 61-69 (1998).

${ }^{24}$ M. Zaider and H. I. Amols, "A little to a lot or a lot to a little: Is NTCP always minimized in multiport therapy,' Int. J. Radiat. Oncol., Biol., Phys. 41, 945-950 (1998).

${ }^{25}$ A. Niermierko, "Reporting and analyzing dose distributions: A concept of equivalent uniform dose,'” Med. Phys. 24, 103-110 (1997).

${ }^{26}$ J. Stein, R. Mohan, X. H. Wang, T. Bortfeld, Q. Wu, K. Preiser, C. C. Ling, and W. Schlegel, "Number and orientations of beams in intensitymodulated radiation treatments,', Med. Phys. 24, 149-160 (1997).

${ }^{27}$ T. Bortfeld, J. Stein, and K. Preiser, 'Clinically relevant intensity modulation optimization using physical criteria,', in Ref. 19, pp. 1-4. 